\title{
Tuberculous Lymphadenitis coexists with Non-Hodgkin Lymphoma
}

\author{
Nenci Siagian, Bramantono ${ }^{2 *}$, Usman Hadi \\ ${ }^{1}$ Department of Internal Medicine, Faculty of Medicine, Universitas Airlangga - Dr. Soetomo General Hospital Surabaya, \\ Indonesia. \\ ${ }^{2}$ Division of Tropical Infection Disease, Department of Internal Medicine, Faculty of Medicine, Universitas Airlangga - Dr. \\ Soetomo General Hospital Surabaya, Indonesia.
}

\begin{abstract}
A B S T RA C T
Tuberculous Lymphadenitis (TBLN) is most common extrapulmonary tuberculosis. The common symptom of TBLN is cervical lymphadenopathy which is known to mimic numerous pathological conditions like NHL. Coexistency TBLN and lymphoma is a rare. A woman, 56 years old, had chief complaint of cervical masses since 2 months ago. She had history of weight loss, fever and night sweats but no history of chronic cough. From physical examination and supporting examination, the patient was diagnosed with TBLN coexists with NHL. She got antituberculosis drug (ATD) for 2 weeks before chemotherapy. The patient died of septic shock 9 days later after chemotherapy. From HPE examination, TBLN and NHL may show simillar feature so Zhiel-Neelsen staining and Immunohistochemical are important to confirm each disease. ATD was given to supress the mycobacterium activity before chemotherapy. However the patient had febrile neutropenia after chemotherapy and died of septic shock. Both TBLN and NHL may occur with simillar sign and symptom and HPE. Further examinations have to be done to confirm the diagnosis of both disesases. Although ATD had given to prevent Tb infection progresivity. On 7 days after chemotherapy she had febrile neutropenia and lead to death due to septic shock.
\end{abstract}

Keywords: Tuberculous lymphadenitis, Non-hodgkin lymphoma, Chemotherapy

Correspondence: Bramantono

E-mail: ibambramantono@gmail.com

Article history: • Received 18 August $2020 \bullet$ Received in revised form 28 August 2020•Accepted 31 August 2020

\section{INTRODUCTION}

Extrapulmonary tuberculosis (TB) accounts for 10$50 \%$ of all TB in human immunodeficiency virus (HIV) negative patients and approximately $35-80 \%$ in HIV infected patients. Tuberculous Lymphadenitis (TBLN) is one of the most common extrapulmonary manifestations of tuberculosis in both HIV-seronegative and HIV-infected patients $(35 \%$ of cases worldwide). TB lymphadenitis occurs more frequently in women than in men. The common symptom of TBLN is cervical lymphadenopathy $(63-77 \%)$ and a high index of suspicion is needed for diagnosis which is known to mimic numerous pathological conditions (Lapausa, et al., 2014; Raviglione, M.C., 2015).

Non-hodgkin's Lymphoma (NHL) is a cancer that starts in white blood cells called lymphocytes, which are part of the body's immune system and helps fighting infections and some other diseases. In the United State, the prevalence of NHL is about $4 \%$ of all cancers and more than half of patients are 65 years old or older at the time of diagnosis and more frequent in men. Lymphomas can start anywhere in the body where lymph tissue is found including lymph nodes, spleen and bone marrow, thymus, denoids and tonsils and digestive tract (Rinaldi, et al., 2014)

Coexistency presentation of TBLN and lymphoma is a rare. Immunosupression is the main cause of mycobacterial infection in Hodgkin's disease and tuberculosis is the main cause of mortality in such case. However, missdiagnose or delay in diagnosis of both TBLN and NHL may occur because of similar signs and symptoms like cervical lymphadenopathy, fever, loss of appetite, loss of weight and night sweats. Thus as a clinician, it's very important to know how to diagnose and treat TBLN with NHL. We report a case of TBLN coexists with NHL in the same organ that ilustrates the management of the diagnose and treatment (Raviglione, M.C., 2015; Rinaldi, et al., 2014).

\section{CASE REPORT}

A woman, 56 years old, a Javanese, a housewife, was admitted to our medical center with chief complaint of cervical masses. Two months berfore her admission, single mass first developed on the left cervical, it was about 1-2 $\mathrm{cm}$, non-tender and painless. While 1 month later the mass became multiple and occured on another side, with various size and became tense and pain. She had history of lethargy, weight loss due to low intake, fever and night sweats since one month before admission. There was no complaint of urinary and bowel habbit. She had been treated with anti tuberculosis drug (ATD) for 2 weeks before admitted. She had no personal and nor family history of TB. The patient 
also had no history of chronic cough, diabetes melitus, hypertension and malignancy before admitted.

From physical examination we found the performance status of the patient was good with temperature (T) was $38^{\circ} \mathrm{C}$ and pain scale was 1-2. From head and neck examination, we found pale conjunctiva. We got enlargement of peripheral lumph nodes in various size on the both side of cervical. On the submandibula sinistra: multiple, \pm 1.5 $2.5 \mathrm{~cm}$, non tender, warm and pain, on the supraclavicula and colli sinistra region: multiple, $\pm 3-4 \mathrm{~cm}$, non tender, warm and pain; or the submandibula dextra regio: single, $\pm 2-3 \mathrm{~cm}$, non tender, warm and pain. Chest examination and abdomen examination were within normal limit. On extrimities examination, we got multiple enlargement of left inguinal lymph nodes, $\pm 2-3 \mathrm{~cm}$, non tender, pain.

Laboratory examination showed $\mathrm{Hb} 7,2 \mathrm{~g} / \mathrm{dL}, \mathrm{RBC}$ $2.74 \times 106 / \mu \mathrm{L}$, HCT 21.8\%, MCV $79.6 \mathrm{fL}, \mathrm{MCH} 26.3$ pg, MCHC 33 g/dL, WBC 10300/ $\mu \mathrm{L}$, Neut 51.5\%, Lymp $21.5 \%$, PLT $234000 / \mu \mathrm{L}$, SGOT $66.0 \mathrm{U} / \mathrm{L}$, SGPT $88.0 \mathrm{U} / \mathrm{L}$, Albumin $3.0 \mathrm{~g} / \mathrm{dL}$, renal function and electrolite serum were within normal limit, HIV rapid test, HbsAg and AntiHCV were non-reactive. Chest X-ray: Cor and Pulmo were within normal limit. Upper and lower abdomen ultrasound: enlargement of para aorta lymph node. Musculoskeletal ultrasound 1-side: multiple enlargement of lymph nodes at left submandibular, left supraclavicular, left cervical, and left inguinal. Histopathology examination (HPE) of left cervical lymph node: NHL, large B cell type, high grade, coexistences with granulomatous lymphadenitis tuberculosa (Ziehl-Neelsen staining was positive acid fast bacilli). Immunohistochemistry showed CD20 positive, $\mathrm{Ki}$ 67 positive (90\%).

Based on the data, the patient was diagnosed with NHL colli, inguinal, para aorta, stadium IIIB, diffuse large B cell type (DLBC), high grade with TBLN, anemia, and hypoalbuminemia. We continued examination including gen expert, erytrocyte sedimentation rate, LDH, blood smear analysis pre blood transfusion and followed $\mathrm{Hb}$ level evaluation post transfusion. The patient was given high calories high protein diet $2100 \mathrm{kcal} /$ day (with extra egg whites) and fluid support with aminofusin $500 \mathrm{ml}$ and D10 $500 \mathrm{ml} /$ day, blood transfusion with pack red cell (PRC) $1 \mathrm{ml} / \mathrm{kg} /$ hour until $\mathrm{Hb} \geq 10 \mathrm{~g} / \mathrm{dL}$ (max. 2 bags/day), paracetamol $500 \mathrm{mg} / 8 \mathrm{~h}$ per oral if the temperatur $>37.5^{\circ} \mathrm{C}$ or pain, ATD with FDC (Rifampycin 150mg, Isoniazid $75 \mathrm{mg}$, Pirazynamid 400mg, Ethambutol 275mg).

\section{Disease Progression}

On the $3^{\text {rd }}$ day of treatment, the symptom of anemia improved. Vital sign was stable and the pain score decreased. The enlargement of cervical lymph nodes was the same. Laboratory results showed erythrocyte sedimentation rate was $82 \mathrm{~mm} / \mathrm{h}, \mathrm{Hb}$ was $10.2 \mathrm{~g} / \mathrm{dL}$, peripheral blood smear showed erythrocyte normositer, hypochromic, without normoblast. Gen expert could not be performed because the patient objected to have re-open biopsy. The patient was prepared to receive chemotherapy with single dose of Rituximab 600mg, Cyclophosphamide $1000 \mathrm{mg}$, Vincristine 2mg, Doxorubicin $80 \mathrm{mg}$ (RCHO) and prednisone $60 \mathrm{mg} /$ day for 3 days.

On the $6^{\text {th }}$ day of treatment (day 2 post chemotherapy) the mass on the left cervical regressed but the performance status decreased and the fever occured intermittenly. The enlargement of cervical lymph on the left cervical: less than $1 \mathrm{~cm}$; on the right cervical: less than $1 \mathrm{~cm}$ and on the submandibular: less than $1 \mathrm{~cm}$. Laboratory results after chemotherapy showed $\mathrm{Hb} 11.0 \mathrm{~g} / \mathrm{dL}$, WBC $6,400 / \mu \mathrm{L}$, Neut: $89.7 \%$, PLT $161,000 / \mu \mathrm{L}$, the rest were within normal limit. The patient was given high calories high protein diet $2,100 \mathrm{kcal} /$ day and fluid therapy with aminofusin $500 \mathrm{ml}$ and D10 $500 \mathrm{ml} /$ day, paracetamol $500 \mathrm{mg} / 8 \mathrm{~h}$ per oral if the temperatur $>37.5^{\circ} \mathrm{C}$ or pain. The patient was under observation for side effect of chemotherapy.

On the day 11 th of treatment (day 7 post chemotherapy): the patient had high fever and became very weak. Physical examination showed GCS 456, blood pressure 100/60 $\mathrm{mmHg}$, pulse $112 \mathrm{bpm}$, regular. Respiratory rate: $22 \mathrm{x} /$ minute, axillar temperature: $38.5^{\circ} \mathrm{C}, \mathrm{SpO}_{2} 99 \%$ without $\mathrm{O}_{2}$ support. Laboratory results showed neutropenia and thrombocytopenia (WBC 320/ $\mu$ L, Neut: $6.2 \%$, ANC: 20 , PLT $82,000 / \mu \mathrm{L}$ ) and hypertransaminasemia (SGOT 155, SGPT 285). Based on the data, in the diagnosis we add sepsis and side effect of chemotherapy (febrile neutropenia $\&$ transaminitis). We planned the patient to have blood and urine culture (anaerobe/aerobe \& fungi) and serial complete blood count. The patient was given the same diet and fluid therapy with normal saline $0.9 \% 2000 \mathrm{ml} / 24 \mathrm{~h}$, injection levofloxacin $750 \mathrm{~g} / 24 \mathrm{~h}$ intravenous, injection ceftazidime $500 \mathrm{mg} / 8 \mathrm{~h}$ intravenous, injection Filgastrim rh G-CSF $300 \mu \mathrm{g} 1$ /day subcutaneous and paracetamol $500 \mathrm{mg} / 8 \mathrm{~h}$ per oral if the temperatur $>37.5^{\circ} \mathrm{C}$.

On the day 13 th of treatment (day 9 post chemotherapy): Physical examination showed GCS 225, Blood pressure was $90 / 50 \mathrm{mmHg}$, Pulse was $124 \mathrm{bpm}$, regular. Respiratory rate was $24 \mathrm{x} /$ minute, the temperature was $39^{\circ} \mathrm{C}, \mathrm{SpO}_{2} 99 \%$ with $\mathrm{O}_{2}$ nasal $3 \mathrm{lpm}$. Laboratory results showed that neutropenia with thrombocytopenia did not improve (WBC $240 / \mu \mathrm{L}$, Neut: $8.3 \%$, ANC: 20 , PLT 32,000/ $\mu \mathrm{L}$ ) and procalcitonin was 114. Based on the data, in the diagnosis we add septic shock due to side of chemotherapy (febrile neutropenia). The patient was given fluid resucitation with normal saline $0.9 \%$ and vasopresor with norepineprin and continue the rest therapies. The patient died of septick shock on day fourtenth of treatment.

\section{DISCUSSION}

TBLN or lymph node TB is considered a local manifestation of the systemic disease. It may occurs during primary TB infection that is initially exposed to tubercle bacilli then multiplies in the lung and then the lymphatic drains the bacilli to hilar lymph nodes (primary complex) and spreads to regional nodes. The most common mode of development of TBLN is post primary disease due to reactivation of dormant foci on the lung parenchyma or reinfection, and progressive primary TB may spread by blood or lymphatics causing extrapulmonary TB (Mahopatra, R.P. and Janmeja, K.A., 2009; Raviglione, et.al., 2015).

M. tuberculosis (MTB) which has bacilli form, acid-fast, growing slowly and UV sensitive, usually enters the human body via the respiratory tract and forms primary complex or Ghon's complex in the posterior segment of the upper 
lobe and undergoes lymphohematogenous dissemination. Because the entry is via respiratory tract, the first lymphoid tissues encountered are presumably hilar and mediastinal lymph nodes. TBLN most frequently involves the cervical lymph nodes followed in frequency by mediastinal, axillary, mesenteric, hepatic, portal, perihepatic and inguinal lymph nodes. The systemic symptoms are not common in TBLN; fever has been reported in $20-50 \%$ of cases in HIVseronegative patient and $60-80 \%$ in HIV-infected patient. The other systemic symptoms that may be found in general extrapulmonary TB are weakness (35\%), anemia (42\%), and lymphopenia (26.4\%) (Mahopatra, R.P. \& Janmeja, K.A., 2009; Raviglione, et.al., 2015).

It presents as unilateral painless swelling of the lymph nodes, most commonly at posterior cervical and supraclavicular sites, it represents $63-77 \%$ of the cases and bilateral disease is uncommon ( $26 \%$ of the cases). In initial stage, TBLN usually shows marked hyperemia, swelling, necrosis, and caseation of the center of the nodes. Then develops into a matted non-tender mass over time and may result in ulceration, fistulous tract draining caseous material or abscess formation. Associated pulmonary disease is present in fewer than $50 \%$ of cases and systemic symptoms are uncommon except in immunocompromised patients (Mohapatra and Janmeja, 2009; Raviglione, et.al., 2015).

The diagnosis can be established by (1) Fine-needle Aspiration Biopsy (FNA) (with a yield up to 80\%). Caseating granuloma with acid-fast bacilli (AFB) positivity is fairly sensitive and spesific for the diagnosis of TB (85\%). However, necrosis which can mimic "caseation" along with granuloma can be seen in many diseases such as lymphoma and sarcoidosis. Histologic features, such as nonspesific lymphoid infiltrates, noncaseating granulomas, or Langerhans giant cells in areas of extensive caseous necrosis, support diagnosis of probable tuberculosis. FNA and Mantoux test were able to diagnose $90 \%$ of the cases (Anmari et al., 2003; Raviglione et al., 2015); (2) Surgical Excision Biopsy, is preferred over incisional biopsy, the latter may be associated with sinus tract formation. Surgical biopsy-excision of the lymph node is the best examination for diagnostic confirmation with sensitivity of $100 \%$ for histological analysis and $60-90 \%$ for the bacilli culture (Fontanilla et al., 2011; Banjelloun A., et al., 2015); (3) Bacteriologic confirmation is achieved in the vast majority of cases, granulomatous lesion or without visible AFBs are typically seen, and cultures are positive in $70-80 \%$ of cases. However, the negative culture does not necessarily exclude the diagnosis because not all enlarged lymph nodes necessarily contain live bacilli. Rapid culture methods have enabled a positive identification method and drug sensitivity within short period (2 to 3 weeks) compared to 8 to 10 weeks by traditional methods (Anmari et al., 2003; Raviglione et al., 2015); (4) Molecular Diagnosis or Nucleic Acid Amplification (NAA) is used to detect mycobacterial DNA instead of detection of Mycobacteria and drug resistance earlier compared to conventional methods. However studies on NAAT for TBLN produce high variable of sensitivity (2-100\%) and specificity (28-100\%), precluding the determination of clinically meaningful estimates of accuracy. (Daley et al., 2007); (5) Ultrasound (USG) and CT scan are known to be effective for detecting enlarged lymph nodes and widely used to obtain information about the size of lymph nodes, localization and accessing their relation wiseventhh major structure in the neck. They are also useful in guidance for biopsy aspiration (Gupta, et al., 2007; Anmari, et.al., 2009).

As the mycobacteria are not documented in every case, certain histopathologic changes are accepted as suggestive of TB. These include granulomatous inflammation with caseation necrosis. The condition including variety of infectious conditions, neoplastic diseases such as lymphomas or metastatic carcinomas, and rare disorders like Kikuchi's disease and Castlema's disease can present the similar cytology or histopathology as TBLN (Gupta, et al., 2007; Raviglione, M.C. et al., 2015).

NHL is one of malignancies of lymphoid cell and is separated from Hodgkin's lymphoma by recognition of Sternberg-Reed cells earlier in the 20th century. About 70,800 new cases of NHL are diagnosed in the United States and nearly 360,000 cases worldwide with various subtypes differ geographically. A number of environmental factors has been implicated in the occurrence of NHL, including infectious agents, chemical exposures and medical treatments. All lymphoid cells are derived from a common hematopoietic progenitor that gives rise to lymphoid, myeloid, erythroid, monocyte, and megakaryocyte lineages. The cell first becomes committed to the lymphoid lineage and then gives rise to B and T cell. About $90 \%$ of all lymphomas are of B-cell origin, and diffuse large B-cell lymphoma (DLBCL) is the most common form of adult levels. NHL comprise a heterogenous group of tumors that differ at the clinical, pathological, molecular and biological (Longo, L.D., 2015).

The sign and symptoms of NHL include weight loss $>10 \%$ in 6 months, temperature $>38^{\circ} \mathrm{C}$ more than 1 weeks, night sweating, weakness, loss of appetite, painless lymph nodes enlargement and liver/spleen enlargement. Laboratory examination may be needed to determine other disease that may coexistent with lymphoma like autoimmune disease (SLE, Sjorgen), hematologic disorder (anemia, leukemia), infectious disease (toxoplasma, tuberculosis) and HIV (Rinaldi, et.al., 2014).

The guideline of NHL by Minisrty of Health of Indonesia recommendation for the diagnostic evaluation of NHL 2014 are as follows: (1) Excisional or core biopsy. The biopsy can be done from the most representative and superficial lymph node. The lymph node on the cervical and supraclavicular are common recommended for biopsy following axilla lymph nodes and the last is inguinal lymph node. Histopathology, immunohistochemical and EBV molecular test should be checked from biopsy specimen. The diagnosis can not be confirmed only by histopathology result, because sometime biopsy is hard to perform. Thus, the combination of biopsy and immunohistochemical may be enough for diagnosis. The histopathology features may show the subtype of NHL based on WHO classification 2016. Lymphoid tumor panels like CD20 presents in the most common types of B-cell lymphomas $(\sim 100 \%)$ and Ki67 is marker for determining the growth fraction and tumor progression; (2) Laboratory. This examination include routine (complete blood count, erythrocyte sedimentation rate, peripheral blood smear, liver function 
test, renal function test, serum electrolite and virus marker urine) and specific laboratory (Electrophoresis protein, Coombs test, B2 microglobulin); (3) Bone marrow aspiration or biopsy; (4) Radiology. CT scan thorax/ abdomen is routine examination for NHL. If it is possible, PET CT scan is more recommended (Rinaldi, et al., 2014; El-Esawy, et al., 2015).

After completing the test and confirm the diagnosis of NHL, we have to do staging that based on AnnArborr staging system which including: (1) Stage 1 - the enlargement of lymph node only at 1 region, either above or below diaphragm; (2) Stage 2- 2 or more lymph node regions are affected, both either or below, but just on one side of the diaphragm; (3) Stage 3 - the lymph node enlargement on both sides of the diaphragm, above and below; (4) Stage 4- the lymphoma has spread beyond the lymphatic system and is now present in both lymph nodes and organs or bone marrow. Health professionals also add the letter " $A$ " or " $B$ " to the stage to indicate the presence of systemic manifestation. The testing also help clinicians to decide the "grade" of the lymphoma. There are 2 main grades of NHL: (1) low-grade or indolent NHL is where the cancer grows slowly, no symptoms for many years; (2) High grade or aggresive NHL is where the cancer grows quickly and aggresively (Rinaldi, et.al., 2014).

In this case, the patient is a female and more than 50 years old. She had multiple lymphnodes enlargement on the both sides of cervical with various size and became tense with pain since two months before admitted. She had history of of lethargy, weight loss, fever, and night sweats since 1 months before admitted. She had been treated with ATD for 2 weeks before admitted. Physical examination showed axilla temperature $38{ }^{\circ} \mathrm{C}$, anemic conjunctiva, various enlargement of peripheral lymph nodes on the submandibular and on the both sides of supraclavicular and inguinal. Laboratory results showed anemia and hypoalbuminemia. Upper and lower abdomen ultrasound: enlargement of para aorta lymph node. Musculoskeletal ultrasound 1-side: multiple enlargement of lymph nodes at left submandibular, left supraclavicular, left cervical, and left inguinal. HPE of left cervical lymph node: NHL, large B cell type, high grade, coexisten with granulomatous TBLN (Ziehl-Neelsen staining was positive acid fast bacilli). Immunohistochemistry showed CD20 positive, Ki 67 positive $(90 \%)$. Based on the data the patient was diagnosed with NHL colli, inguinal, para aorta, stadium IIIB, DLBC, high grade with TBLN on ATD, anemia and hypoalbuminemia.

Concomitant presentation of TB and lymphoma is a rare entity. Very few case reports of such presentation are published till date. Of importance, TB and lymphoma can be causatively related, though the well-established lymphoma related immunosupressant and TB are chronic infection diseases whose presentation and reactivation are known to be promoted by cell mediated immunodeficiency. Then an interesting question arises: Which comes first? Is it the NHL that develop over TBLN or vice versa? Some studies showed that the risk of NHL is increased in individual with history of TB. On the other hand, the incidence of TB in NHL patients is much higher than in the general population. However, no experimental data are present to prove the claim. Ever since biopsy is the most specific and sensitive diagnostic procedure for both NHL and TBLN, but caseating or necrotizing granulomas typical for TB can be found in NHL. Therefore, the definite proof of TB is a positive culture for MTB, or the presence of AFB in biospsy specimen with Zheil-Neelsen staining. CT scan examination could be useful in differentiating TBLN and lymphoma. Peripheral enhancement, frequently with multilocular appearances, was common in TBLN, but was homogenous in lymphomas (Fanourgiakis, et al., 2008, Chen, J., et al., 2012).

Based on the guideline of TB management by Association of Pulmonologist in Indonesia, TBLN is treated like pulmonary TB with Rifampicin, Isoniazid, ethambutol, and pyrazinamide for 2 months and followed with Rifampicin and Isoniazid for 10 months. Based on the guideline of NHL by Ministry of Health of Indonesia, NHL can be treated with rituximab, cyclophosphamide, doxorubicin, vincristine and prednisone (Rinaldi, et.al., 2014; PDPI, 2015).

In this case, the development of NHL in organs previously seeded with MTB resulting in the reactivation of the latent $\mathrm{TB}$ seems possible. The diversity in the immunocompromised status in the patient makes it challenging to common strategy for treatment of latent TB. Retrospective study by Fujita, et al. showed that the TB could be the reason for the delay in cancer treatment and the anti-cancer therapy was restarted after at least 2 weeks, when the infectivity is considered to be decreased. The study result showed that anti-cancer chemotherapy was not a big deal in latent TB. Dres, et al., 2012 reported one case of a 60-year-old man that showed TBLN at mediastinal and retroperitoneal hide lymphoma. The signs and symptom did not improve after 2 weeks anti-tuberculosis and splenectomy was performed because of extensive caseous necrosis and anti-cancer chemotherapy was started too. The patient died of septic shock shortly after. Another case report by Fanourgiakis et al. showed a70-year-old woman with TBLN. The enlargement of lymph nodes failed to regress after anti-tuberculosis therapy for unknown period and then it was known that she had NHL confirmed by complete excision of the involved lymph nodes. The patient died due to massive relapse of the cervical masses in few weeks after chemotherapy (Fanourgiakis, et al., 2008; Dres, et al., 2012; Fujita, et al., 2014).

Approximately $40-50 \%$ of aggressive noncontagious stage III NHL are cured with standard therapy. Scoring system such as the International Prognostic Index (IPI) score have been developed and validated to estimate the response rate or survival rate of the patient. However the chemotherapy side effect including persistent neutropenia in this case should be considered that it could affect the outcome of therapy. Neutropenic sepsis is a potentially fatal complication of chemotherapy. Mortality rates ranging between $2-21 \%$ have been reported in adult. Vakkalanka \& Link, 2011 reported neutropenic sepsis occurred in 2-12\% cases of NHL that had Cyclophosphamide, Doxorubicin, Vincristine, and Prednisone (Simmons, T., 2012). The patient in this case had IPI score 2 points (elevated LDH serum and stage III disease) that indicated low-intermediate 
risk and had been taking ATD since 2 weeks. Side effect of chemotherapy (neutropenia and thrombocytopenia) occurred a week after chemotherapy and 3 days later the patient died.

\section{CONCLUSION}

Case of a 56-year old woman with TBLN coexistence with NHL has been reported. The diagnosis of both diseases was confirmed by excision biopsy to performed histopathology, AFB (Zeihl-Neelsen stain) and histochemical examination. Patient was treated with ATD for 2 weeks to prevent progresivity of TB infection before chemotherapy. Side effect of chemotherapy, febrile neutropenia, ocurred in a week after chemotherapy although the enlargement of masses regressed and 3 days later the patient died because of septic shock.

\section{CONFLICT OF INTEREST}

The author declare there is no conflict of interest of this study.

\section{REFERENCES}

Anmari, F. F., Habi, B. H. A. \& Ghariebeh, I. K. 2003. Tuberculosis of the lymph Glands of the Neck: A Limited Role for Surgery. SAGE Journals. 575579

Banjelloun, A., Darouassi, Y., Zakaria, Y., Bouchentouf, R. \& Errami, N. 2015. Lymph nodes tuberculosis: a retrospective study on clinical and therapeutuc features. Panafrican Medical Journal.

Chen, J., Yang, Z. G., Shao, H., Deng, W., Wen, L.Y. \& Tang, S. S. 2014. Differentiation of Tuberculosis from Lymphomas in Neck Lymph nodes with Multidetector-row computed tomography. Int J Tuberc Lung Dis, 16(12):1686-91

Daley, K. P., Thomas, S. \& Pai, M. 2007. Nucleic Acid Amplification Tests for the Diagnosis of Tuberculous Lymphadenitis: A systemic review. The International Journal of Tuberculosis and Lung Disease., 11(11):1166-76

El-Esawy, B. H., Alghamdy, A. N. \& El Askary, A. 2015. Clinicopathologic Correlation of Ki67 Proliferative Indices among WHO subtypes of Non-Hodgkin's Lymphoma \& it's prognostic Significance.
Res J Medicine \& Med Sci, 10 (1): 1-6

Fanougiakis, P., Mylona, E., Androulakis, I., Eftychiou, C., Vryonis, E., Georgala, A., Skoutelis, A. \& Aoun, M. 2008. Non-Hodgkin's Lymphoma and Tuberculosis Coexistence in the Same Organs: A report of two cases. Postgrad Med J, 84:276-277

Fontanilla, J. M., Barnes, A. \& von Reyn, C. F. 2011. Current Diagnosis and Management of Peripheral Tuberculous Lymphadenitis. Clin Infect Dis.; 53(6):555

Fujita, T., Endo, M., Gu, Y., sato, T. \& Ohmagari, N. 2014. Mycobaterium Tuberculosis Infection in cancer patients at a Tertiary Care Cancer Center in Japan. J infect Chemother, 20:213-216

Gupta, B. K., Kumar, A., Sen, R., Sen, J. \& Verma, M. 2007. Role of Ultrasonography and Computed Tomography in Complicated Cases of Tuberculous Cervical Lymphadenitis. Indian J Tuberc; 54:71-78

Longo, L. D. 2015. Lymph node Tuberculosis. In: KASPER, D. L. (ed.) Harrison's Principles of Internal Medicine,. 19th ed. New York: McGraw Hill Education

Lapausa, M. R., Saldana, M. A. \& Asensio, N. A. 2014. Extrapulmonary Tuberculosis: on overview. Rev Esp Sanid Penit. 17:3-11

Mohapatra, R. P. \& Janmeja, K. A. 2009. Review Article: Tuberculous Lymphadenitis. Journal of the Association of Physicians of India. Vol.57:585-590

Raviglione, M. C. 2015. Lymph node Tuberculosis. In: KASPER, D. L. (ed.) Harrison's Principles of Internal Medicine,. 19th ed. New York: McGraw Hill Education

Rinaldi, I., Hardjolukito, S. R. E., Prajogi, B. G., Giselvania, A., Nuhonni, A.S., Indriani, Pratii, H. B. K., Dewi, L. F., Witjaksono, F., Manikam, M. R. N., \& Octovia, I. L. 2014. Panduan penatalaksanaan Limfoma Non-Hodgkin. Komite Penanggulangan Kanker Nasional- Kementrian Kesehatan Republik Indonesia

Simmons, T. 2012. Prevention and Management of Neutropenic Sepsis in Cancer Patients: Full needs assessment report. The National Institute for Health Clinical Excellence (NICE 